deliberations that have taken place over the last three years.

\section{The Concept of the Global Ocean Observing System (GOOS)}

The objective of the GOOS is a permanent system of global and systematic observations adequate for forecasting climate variability and change: for assessing the health or state of the marine environment and its resources, including the coastal zone; and for supporting an improved decision-making and management process, which takes into account potential natural and man-made changes in the environment and their effects on human health and resources.

The GOOS is an internationally coordinated system for systematic operational data collection (measurements), data analysis. exchange of data and data products, technology development, and transfer. GOOS will use a globally coordinated. scientifically based strategy to allow for mon- itoring and subsequent prediction of environmental changes globally, regionally. and nationally. Data will be generated by repeat sampling and remote sensing using sea-surface and subsurface instrumentation in the open sea and in coastal regions worldwide. including enclosed and semienclosed areas. Major physical. chemical, and biological variables have been identified by the HOTO Panel that can be used to provide an integrated assessment of the current health of the oceans and early warning of deterioration.

Guided by IOC, WMO, UNEP, and the International Council for Scientific Unions (ICSU), the planning for GOOS is taking place within five specific areas:

- climate monitoring. assessment, and prediction:

- monitoring and assessment of living resources:

- monitoring the coastal environment and its changes:
- assessment and prediction of the health of the ocean; and

- marine meteorological and operational services.

The GOOS is a set of activities that constitutes operational oceanography and its related efforts, including some directed and applied research. GOOS is Oceanography for societal and economic purposes (see Fleming. 1995). coordinating and fostering ocean operations and applications that are long-term, routine, globally relevant, scientifically based, systematic, and affordable.

The GOOS is not a research program but builds on research, it provides data for research, and supporting directed and applied research is a significant contribution to GOOS. Academic institutions have provided the foundation upon which GOOS is built, through research programs. technology development. and training of human resources. A strong

\title{
What DOES GOOS/HOTO MEAN, AND What WiLl IT OFFER, TO THE MARINE SCIENTIST?
}

\author{
By Herbert L. Windom
}

$\mathrm{T}$ HE IMPLEMENTATION of the Health of the Oceans Module (HOTO) of the Global Ocean Observing System (GOOS) is intended to occur. region by region. in concert and ideally amalgamated, with the other GOOS components, particularly the climate, living resources, and coastal zone modules. However, what HOTO potentially offers to the marine scientist is a scheme of continuing measurements accomplished in a more comparable manner. that can be used by individuals for their own applications. The global development of HOTO is through national and regional implementation activities that address identified problems in specific geographic areas and assess their extent in time and space. Individual scientists, therefore, should seize the opportunity of becoming involved in the planning and implementation of such specific monitoring activities. HOTO data will be made available in raw form and in a range of collated and interpreted forms that will be useful for a suite of marine and nonmarine applications. HOTO also will provide a mechanism for obtaining a measurement series on which individual scientists can base, or piggyback, any specific measurement requirements that they have for scientific and/or management applications. This then is the promise of HOTO. It is now up to the community involved in the design of the several GOOS modules to complete their work and to use the international science, environmental management, and policy communities to ensure the implementation of all the modules in a systematic and coordinated manner. The entire sequence of operations within GOOS, if implemented as intended, will provide a vastly improved store of basic marine environmental data with higher spatial and temporal resolution than available ever before. This will mean that individual scientists can reduce the effort they have to devote to routine measurements and concentrate their efforts on the necessary incremental measurements for their own purposes.

Herbert L. Windom, Skidaway Institute of Oceanography. Savannah, Georgia 31411. USA. 